\title{
Trace and contextual fear conditioning require neural activity and NMDA receptor-dependent transmission in the medial prefrontal cortex
}

\author{
Marieke R. Gilmartin ${ }^{1}$ and Fred J. Helmstetter \\ Department of Psychology, University of Wisconsin-Milwaukee, Milwaukee, Wisconsin 53211, USA
}

\begin{abstract}
The contribution of the medial prefrontal cortex (mPFC) to the formation of memory is a subject of considerable recent interest. Notably, the mechanisms supporting memory acquisition in this structure are poorly understood. The mPFC has been implicated in the acquisition of trace fear conditioning, a task that requires the association of a conditional stimulus (CS) and an aversive unconditional stimulus (UCS) across a temporal gap. In both rat and human subjects, frontal regions show increased activity during the trace interval separating the CS and UCS. We investigated the contribution of prefrontal neural activity in the rat to the acquisition of trace fear conditioning using microinfusions of the $\gamma$-aminobutyric acid type $\mathrm{A}\left(\mathrm{GABA}_{\mathrm{A}}\right)$ receptor agonist muscimol. We also investigated the role of prefrontal $N$-methyl-D-aspartate (NMDA) receptormediated signaling in trace fear conditioning using the NMDA receptor antagonist 2-amino-5-phosphonovaleric acid (APV). Temporary inactivation of prefrontal activity with muscimol or blockade of NMDA receptor-dependent transmission in mPFC impaired the acquisition of trace, but not delay, conditional fear responses. Simultaneously acquired contextual fear responses were also impaired in drug-treated rats exposed to trace or delay, but not unpaired, training protocols. Our results support the idea that synaptic plasticity within the mPFC is critical for the long-term storage of memory in trace fear conditioning.
\end{abstract}

The prefrontal cortex participates in a wide range of complex cognitive functions including working memory, attention, and behavioral inhibition (Fuster 2001). In recent years, the known functions of the prefrontal cortex have been extended to include a role in long-term memory encoding and retrieval (Blumenfeld and Ranganath 2006; Jung et al. 2008). The prefrontal cortex may be involved in the acquisition, expression, extinction, and systems consolidation of memory (Frankland et al. 2004; Santini et al. 2004; Takehara-Nishiuchi et al. 2005; Corcoran and Quirk 2007; Jung et al. 2008). Of these processes, the mechanisms supporting the acquisition of memory may be the least understood. Recently, the medial prefrontal cortex (mPFC) has been shown to be important for trace fear conditioning (Runyan et al. 2004; Gilmartin and McEchron 2005), which provides a powerful model system for studying the neurobiological basis of prefrontal contributions to memory. Trace fear conditioning is a variant of standard "delay" fear conditioning in which a neutral conditional stimulus (CS) is paired with an aversive unconditional stimulus (UCS). Trace conditioning differs from delay conditioning by the addition of a stimulus-free "trace" interval of several seconds separating the CS and UCS. Learning the CS-UCS association across this interval requires forebrain structures such as the hippocampus and mPFC. Importantly, the MPFC and hippocampus are only necessary for learning when a trace interval separates the stimuli (Solomon et al. 1986; Kronforst-Collins and Disterhoft 1998; McEchron et al. 1998; Takehara-Nishiuchi et al. 2005). This forebrain dependence has led to the hypothesis that neural activity in these structures is necessary to bridge the CS-UCS temporal gap. In support of this hypothesis, single neurons recorded from the prelimbic area of the rat mPFC exhibit sustained increases in firing during the CS and trace interval in trace fear

'Corresponding author.

E-mail marieke.gilmartin@gmail.com; fax (414) 229-5219.

Article is online at http://www.learnmem.org/cgi/doi/10.1101/lm.1597410. conditioning (Baeg et al. 2001; Gilmartin and McEchron 2005). Similar sustained responses are not observed following the CS in delay conditioned animals or unpaired control animals. This pattern of activity is consistent with a working memory or "bridging" role for $\mathrm{mPFC}$ in trace fear conditioning, but it is not clear whether this activity is actually necessary for learning. We address this issue here using the $\gamma$-aminobutyric acid type $\mathrm{A}\left(\mathrm{GABA}_{\mathrm{A}}\right)$ receptor agonist muscimol to temporarily inactivate cellular activity in the prelimbic $\mathrm{mPFC}$ during the acquisition of trace fear conditioning.

The contribution of mPFC to the long-term storage (i.e., $24 \mathrm{~h}$ or more) of trace fear conditioning, as opposed to a strictly working memory role (i.e., seconds to minutes), is a matter of some debate. Recent reports suggest that intact prefrontal activity at the time of testing is required for the recall of trace fear conditioning $2 \mathrm{~d}$ after training (Blum et al. 2006a), while mPFC lesions performed $1 \mathrm{~d}$ after training fail to disrupt the memory (Quinn et al. 2008). The findings from the former study may reflect a role for prelimbic mPFC in the expression of conditional fear rather than memory storage per se (Corcoran and Quirk 2007). However, blockade of the intracellular mitogen-activated protein kinase (MAPK) cascade during training impairs the subsequent retention of trace fear conditioning $48 \mathrm{~h}$ later (Runyan et al. 2004). Activation of the MAPK signaling cascade can result in the synthesis of proteins necessary for synaptic strengthening, providing a potential mechanism by which $\mathrm{mPFC}$ may participate in memory storage. To better understand the nature of the prefrontal contribution to long-term memory, more information is needed about fundamental plasticity mechanisms in this structure. Dependence on $N$-methyl-D-aspartate receptors (NMDAR) is a key feature of many forms of long-term memory, both in vitro and in vivo. The induction of long-term potentiation (LTP) in the hippocampus, a cellular model of long-term plasticity and information storage, requires NMDAR activation (Reymann et al. 1989). Genetic knockdown or pharmacological blockade of NMDAR-mediated neurotransmission in the hippocampus 
impairs several forms of hippocampus-dependent memory, including trace fear conditioning (Tonegawa et al. 1996; Huerta et al. 2000; Quinn et al. 2005), but it is unknown if activation of these receptors is necessary in the $\mathrm{MPFC}$ for the acquisition of trace fear conditioning. Data from in vivo electrophysiology studies have shown that stimulation of ventral hippocampal inputs to prelimbic neurons in MPFC produces LTP, and the induction of prefrontal LTP depends upon functional NMDARs (Laroche et al. 1990; Jay et al. 1995). If the role of mPFC in trace fear conditioning goes beyond simply maintaining CS information in working memory, then activation of NMDAR may be critical to memory formation. We test this hypothesis by reversibly blocking NMDAR neurotransmission with 2-amino-5-phosphonovaleric acid (APV) during training to examine the role of prefrontal NMDAR to the acquisition of trace fear conditioning.

Another important question is whether mPFC contributes to the formation of contextual fear memories. Fear to the training context is acquired simultaneously with fear to the auditory CS in both trace and delay fear conditioning. Conflicting reports in the literature suggest the role of $\mathrm{mPFC}$ in contextual fear conditioning is unclear. Damage to ventral areas of mPFC prior to delay fear conditioning has failed to impair context fear acquisition (Morgan et al. 1993). Prefrontal lesions incorporating dorsal $\mathrm{mPFC}$ have in some cases been reported to augment fear responses to the context (Morgan and LeDoux 1995), while blockade of NMDAR transmission has impaired contextual fear conditioning (Zhao et al. 2005). Post-training lesions of mPFC impair context fear retention (Quinn et al. 2008) in trace and delay conditioning. Contextual fear responses were assessed in this study to determine the contribution of neuronal activity and NMDAR-mediated signaling in $\mathrm{mPFC}$ to the acquisition of contextual fear conditioning.

\section{Results}

The contribution of prelimbic mPFC activity and NMDA receptormediated signaling to the acquisition of trace fear conditioning was examined using pretraining infusions of the $\mathrm{GABA}_{\mathrm{A}}$ receptor agonist muscimol or the NMDA receptor antagonist APV. On the day of training, rats received bilateral infusions of muscimol, APV, or saline vehicle into the prelimbic mPFC $15 \mathrm{~min}$ before trace (Trace saline $n=16$; Trace muscimol $n=13$; Trace APV $n=12$ ) or delay (Delay saline $n=15$; Delay muscimol $n=10$; Delay APV $n=12$ ) fear conditioning, or unpaired control training (Unpaired saline $n=10$; Unpaired muscimol $n=9$; Unpaired APV $n=11)$. All rats were tested for CS and context fear retention $24 \mathrm{~h}$ later, and freezing was used as the measure of conditional fear. During the training session, prefrontal inactivation or blockade of NMDA receptor activity did not impair the expression of freezing. This observation was confirmed by analysis of freezing during the post-acquisition period immediately following trace, delay, or unpaired training. One-way ANOVAs on each training group revealed no effect of drug treatment on post-acquisition freezing in any group (data not shown; $F<1.00, P>0.05$ for each group).

\section{Responding to the auditory CS}

Inhibition of prelimbic activity or specific blockade of NMDARmediated signaling in the mPFC during training impairs the acquisition of trace, but not delay, fear conditioning. Twentyfour hours after training, rats were tested for retention of conditional fear to the CS in a novel chamber. Figure 1 shows the mean percentage of time each group of rats spent freezing during the 6-min pre-CS baseline period and during the 5-min CS presentation during the CS retention test. Freezing during the 4-min post-CS period is not shown in this figure for the purpose of
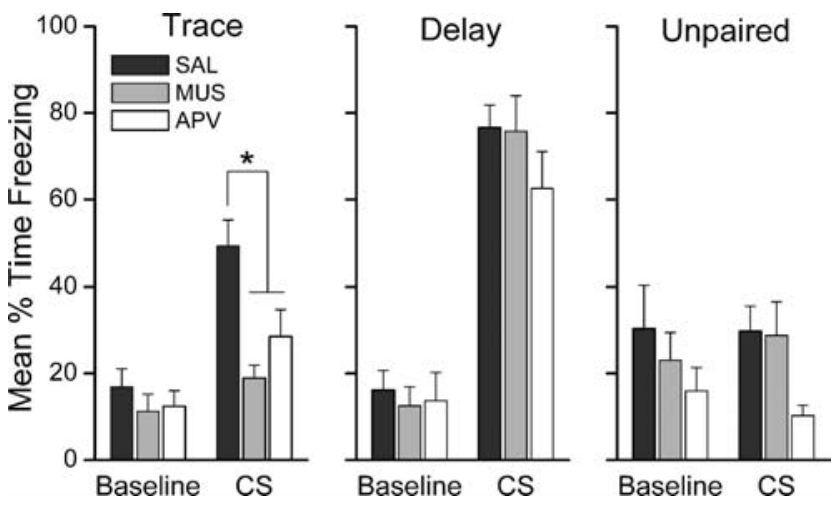

Figure 1. Prefrontal neural activity and NMDA receptors are necessary for the formation of trace fear memory. Graphs show the mean $( \pm S E M)$ percent time spent freezing during the CS retention test $24 \mathrm{~h}$ after training. Saline-infused trace and delay rats showed increased freezing during the CS relative to baseline. Inactivation of mPFC with muscimol or blockade of prefrontal NMDAR with APV prior to trace, but not delay, fear conditioning impaired subsequent retention of fear. Drug-infused TFC rats showed levels of CS freezing similar to unpaired controls. ${ }^{*} P<0.05$.

clarity, but was included in analyses. Figure 2 shows the minuteby-minute mean freezing during the CS retention test, including the post-CS period. Analysis of freezing during the retention test using three separate $3 \times 3$ mixed-model ANOVAs with withinsubjects Period (Baseline, CS, Post-CS) and between-subjects Drug (Saline, Muscimol, APV) factors revealed a significant main effect of Period in each group: Trace $F_{(2,70)}=31.15, P<0.0001$; Delay $F_{(2,68)}=81.41, P<0.0001$; Unpaired $F_{(2,52)}=11.72, P<$ 0.0001 . Follow-up LSD post-hoc analyses on these significant main effects of Period showed that trace and delay conditioned rats froze more during the CS than during the baseline $(P<$ 0.0001 for each group). In contrast, unpaired rats showed no difference in freezing during the baseline and CS periods $(P=$ 0.931). All groups showed greater freezing in the post-CS period compared with baseline (all $P<0.0001$ ). These results show that rats receiving paired, but not unpaired, presentations of the CS and UCS exhibit conditional fear to the CS during retention testing. Disruption of the mPFC during paired conditioning in trace, but not delay, impairs this CS-UCS association. The mixed-model ANOVA for the trace group revealed a significant Drug $\times$ Period interaction, $F_{(4,70)}=2.97 ; P<0.025$. Follow-up LSD post-hoc analysis of this interaction showed that muscimol- and APV-infused rats froze significantly less than saline-infused rats during the CS $(P<0.001, P<0.013$, respectively). Post-CS freezing was similarly impaired in these rats $(P<0.017, P<0.003$, respectively). No significant Drug $\times$ Period interaction was revealed in the analysis of the delay or unpaired rats. Together, these data show that prefrontal activity in general and NMDA receptor activation in the $\mathrm{mPFC}$ are necessary for trace but not delay fear conditioning.

\section{Responding to the training context}

Inhibition of prelimbic activity or blockade of NMDAR-mediated signaling in mPFC during training impairs the acquisition of context fear during auditory-paired fear conditioning. Twenty-four hours after training, rats were also tested for retention of conditional fear to the context in the original training chamber. Figure 3 shows the mean percent of time each group of rats spent freezing during the 15-min exposure to the training context. Trace and delay conditioned rats, but not unpaired control rats, that had received muscimol or APV infusions exhibited decreased freezing 

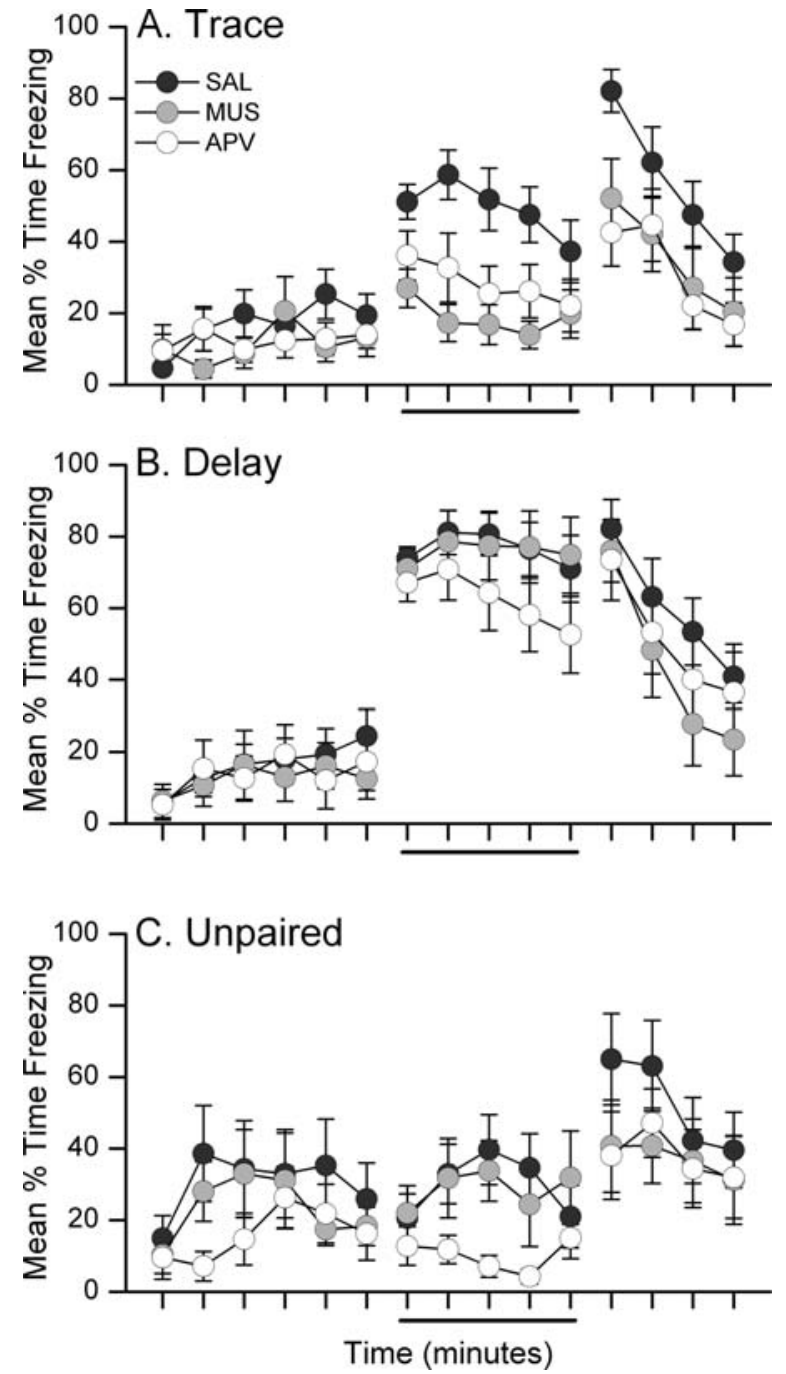

Figure 2. Impairments in trace fear retention in muscimol- and APV-infused rats occur during the CS and post-CS periods of the CS retention test. Graphs show the mean ( \pm SEM) percent time spent freezing during each minute of the 15-min CS retention test for trace conditioned $(A)$, delay conditioned $(B)$, or unpaired $(C)$ control rats. The solid line below the $x$-axis marks the 5 -min presentation of the white noise CS. Unpaired rats showed slightly greater baseline freezing than conditioned rats, but did not show a significant change in freezing during the CS. Unpaired APV rats on average froze less than unpaired saline or muscimol rats, but this difference was not supported with a significant main effect of $\operatorname{Drug}(F<2.00 ; P>0.05)$.

during the context reexposure compared with saline-infused controls. A one-way ANOVA on context freezing in the trace group revealed a significant effect of Drug treatment, $F_{(2,35)}=$ 7.05, $P<0.003$. LSD post-hoc analysis showed that rats in the muscimol and APV conditions froze significantly less than saline control rats $(P<0.002, P<0.007$, respectively). Similarly, analysis of context freezing in the delay group revealed an effect of Drug treatment, $F_{(2,34)}=3.96, P<0.029$. LSD post-hoc analysis showed that rats in the muscimol and APV conditions froze significantly less than saline control rats $(P<0.049, P<0.013$, respectively). In contrast, infusion of muscimol or APV had no effect on contextual fear acquisition in the unpaired group, $F_{(2,26)}=1.78, P=0.189$. This was an interesting finding because the mechanisms supporting contextual fear conditioning were expected to be similar in each training group. However, these data show that general activity and NMDA receptor activation in prelimbic mPFC are necessary for the acquisition of fear responses to the training context in some, but not necessarily all, conditioning paradigms.

\section{Reacquisition of conditional fear responses}

The observed impairments in trace and contextual fear conditioning following pretraining muscimol and APV infusions were not a result of permanent damage to prefrontal tissue. This was confirmed by retraining a subset of trace $(n=29)$ and delay $(n=22)$ rats in the absence of any drug. These rats were tested for CS and context retention $24 \mathrm{~h}$ later in the same manner as the original tests. A comparison of impaired retention in the first test with intact retention after retraining can be found in Figure 4 . Figure 4 shows the minute-by-minute freezing during the CS retention test $24 \mathrm{~h}$ after initial trace conditioning (panel A) and during the CS retention test following retraining (panel B). A $3 \times 3$ mixed-model ANOVA with within-subjects Period (Baseline, CS, Post-CS) and between-subjects Drug (Saline, Muscimol, APV) factors on freezing during this second test revealed a significant main effect of Period, $F_{(2,52)}=17.60, P<0.0001$. Follow-up LSD post-hoc analysis showed that trace rats froze significantly more during the CS and post-CS periods compared with baseline freezing. There was no effect of drug history on freezing as neither the main effect of Drug $\left(F_{(2,26)}=0.31\right)$ nor the Drug $\times$ Period $\left(F_{(4,52)}=0.12\right)$ interaction was significant. The same analysis was applied to the delay group during the CS retention after retraining (data not shown). There was no main effect of Drug nor a Drug $\times$ Period interaction, but the ANOVA revealed a significant main effect of Period $F_{(2,38)}=50.66, P<0.0001$. Follow-up LSD post-hoc analysis of freezing during each period showed that, regardless of drug history, delay rats froze significantly more during the CS and post-CS periods than during baseline. Just as with acquisition of fear to the CS, trace and delay rats were able to acquire contextual fear responses when retrained in the absence of drug. Figure 4 shows the mean time spent freezing during the context retention test $24 \mathrm{~h}$ after initial training (panel C) or $24 \mathrm{~h}$ after retraining (panel D). A one-way ANOVA on each group in panel D did not reach significance, $(F<1.00$ for each group). Together, these findings demonstrate that the effects of muscimol and APV were
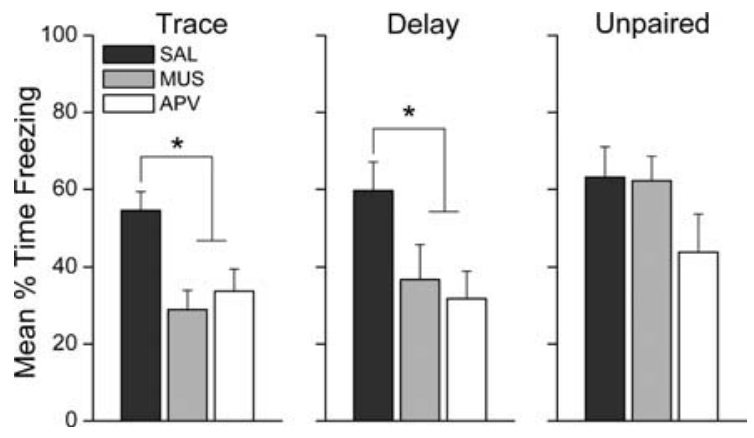

Figure 3. Prefrontal neural activity and NMDA receptors are necessary for the formation of background contextual fear memory. Graphs show the mean $( \pm$ SEM) percent time spent freezing during the 15-min context retention test $24 \mathrm{~h}$ after training. Saline-infused rats in all groups showed robust freezing during the context reexposure. Inactivation of mPFC with muscimol or blockade of prefrontal NMDAR with APV prior to trace, but not delay, fear conditioning impaired subsequent retention of fear. ${ }^{*} P<0.05$. 

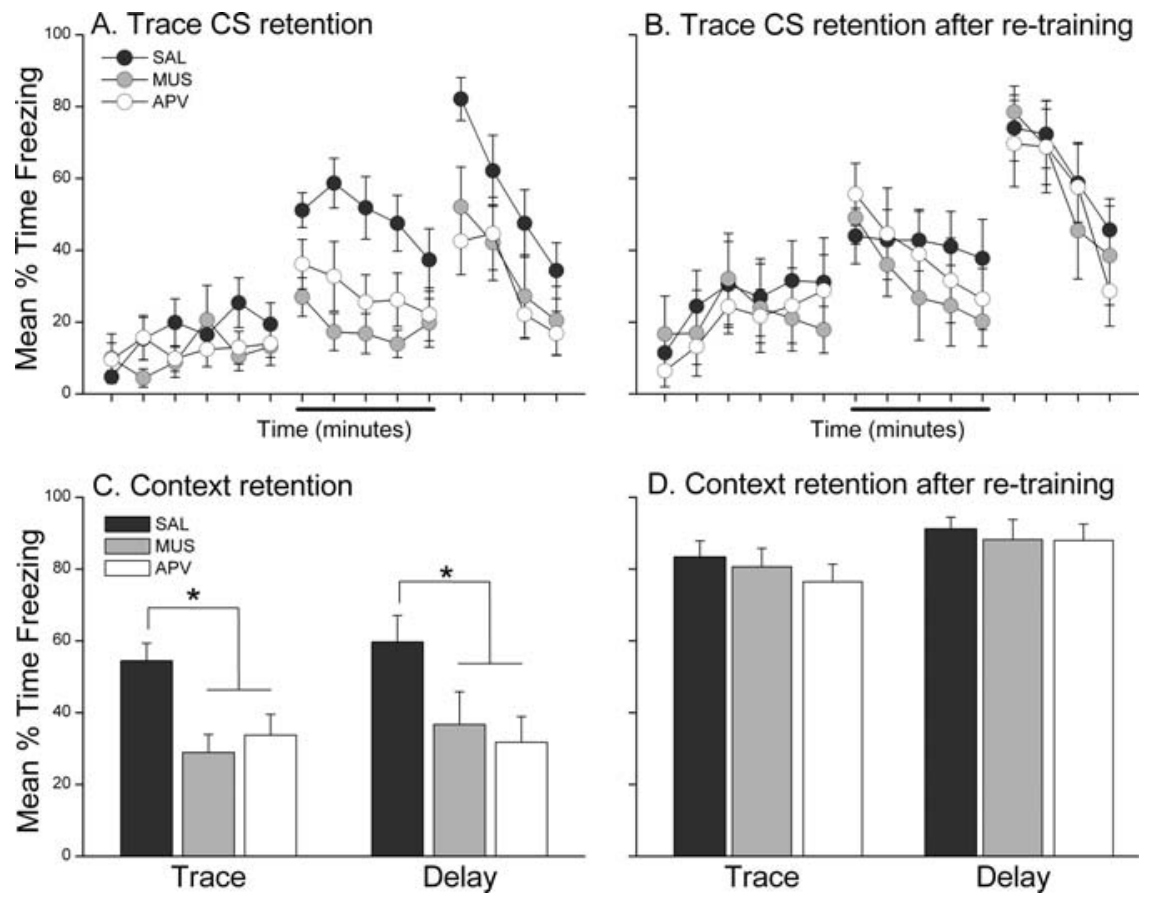

Figure 4. Previously impaired rats were able to acquire trace and contextual fear responses when retrained in the absence of muscimol or APV. Graphs in panels $A$ and $B$ show the mean ( \pm SEM) percent time spent freezing during each minute of the first CS retention test $24 \mathrm{~h}$ after initial training $(A)$ and during each minute of the $C S$ retention test $24 \mathrm{~h}$ after retraining $(B)$. The data in panel $A$ was redrawn from Figure $2 \mathrm{~A}$. When retrained with an intact $\mathrm{mPFC}$, previously impaired muscimol and APV rats showed CS freezing similar to saline control rats. Graphs in panels $C$ and $D$ show the mean $( \pm$ SEM) percent time spent freezing during the 15 -min context retention test $24 \mathrm{~h}$ after initial training $(C)$ and during the 15 -min context retention test $24 \mathrm{~h}$ after retraining $(D)$. The data in panel $C$ was redrawn from Figure 3. Previously impaired muscimol and APV rats in both the trace and delay groups acquired contextual fear responses similar to saline controls when retrained with an intact mPFC. ${ }^{*} P<0.05$.

indeed temporary and that previously impaired rats were able to acquire conditional fear responses to the CS and context when retrained with an intact $\mathrm{mPFC}$.

\section{Histology}

Behavioral analyses were conducted using 108 of the 120 rats in this study. All 120 rats showed reliable bilateral cannula placement in the prelimbic area of the mPFC. Figure 5 shows the location of the guide cannulae placed correctly in the prelimbic area. Twelve rats were excluded from analyses because of extensive tissue damage to the prelimbic beyond the immediate area of the cannula site $(n=10)$, infection $(n=1)$, or blocked cannulae $(n=1)$. This study did not directly test the spread of drug infusion; however, based on previous reports, we estimate the diffusion of drug did not extend substantially beyond the prelimbic region of $\mathrm{mPFC}$. A recent quantitative analysis of the spread of fluorescently labeled muscimol $(1 \mu \mathrm{g} / \mu \mathrm{L})$ in the mPFC showed that an infusion of $0.5 \mu \mathrm{L}$ muscimol in saline vehicle has an effective area between 0.5 and $1.0 \mathrm{~mm}$ around the infusion site with white matter providing natural boundaries to the spread (Allen et al. 2008). Fluorescently labeled muscimol may, however, have a more limited spread compared with unlabeled muscimol because of its higher molecular weight. Autoradiographic analysis of $\left[{ }^{3} \mathrm{H}\right]$ muscimol may provide a better estimation of spread, and the infusion of a larger volume of $1.0 \mu \mathrm{L}\left[{ }^{3} \mathrm{H}\right]$ muscimol into cortex has been shown to have an average maximal spread of $1.7 \mathrm{~mm}$ (Martin 1991).
Furthermore, glucose uptake (a measure of functional inactivation) is maximally reduced within $1.0 \mathrm{~mm}$ of the muscimol infusion site (Martin 1991). A similar diffusion pattern of $1.0 \mu \mathrm{L}$ APV has been reported in hippocampus (Steele and Morris 1999). Using $0.5 \mu \mathrm{L}$ APV in CA3 hippocampus, Lee and Kesner (2002) showed that this size infusion was functionally limited to the CA3 subregion of the hippocampus as LTP was affected in CA3, but not in the neighboring CA1 and dentate gyrus subregions of the hippocampus. We have used a more conservative volume of $0.3 \mu \mathrm{L}$, which has been used successfully to distinguish between prelimbic and infralimbic mPFC function (LaLumiere et al. 2010). While we cannot rule out the possibility that diffusion of muscimol or APV may have extended a small amount into the more ventral IL or the more dorsal anterior cingulate areas of mPFC, we are reasonably confident that the effective area of drug infusion in this study is within the prelimbic mPFC.

\section{Discussion}

This study examined the effects of temporary inactivation of prelimbic $\mathrm{MPFC}$ and blockade of NMDAR-mediated neurotransmission on the acquisition of trace and delay fear conditioning. Both manipulations of prefrontal function during trace fear conditioning impaired retention of fear to the CS and context. In contrast, muscimol and APV infusion had no effect on retention for the delay conditioned CS, but did impair context fear retention in these rats. These results suggest that the acquisition of trace and contextual fear conditioning requires cellular activity in the prelimbic $\mathrm{MPFC}$ and also activation of prefrontal NMDAR receptors.

Disruption of cellular activity in prelimbic mPFC during training impaired subsequent CS retention in the trace, but not the delay groups. This is consistent with previous findings, which showed that prefrontal activity is not necessary for the acquisition of standard delay fear conditioning. Permanent lesions of the ventral and dorsal areas of the mPFC have no effect on the acquisition and retention of delay fear conditioning (Morgan et al. 2003; but see Sacchetti et al. 2002). In contrast, prefrontal lesions impair acquisition and retention of trace eyeblink conditioning, in which an auditory CS is paired with a air puff or periorbital shock UCS separated by a 500-msec trace interval (Kronforst-Collins and Disterhoft 1998; Weible et al. 2000; McLaughlin et al. 2002). Similar impairments in trace eyeblink conditioning were observed using temporary inactivation of mPFC with muscimol during training (Takehara-Nishiuchi et al. 2005). We have extended these findings to trace fear conditioning in which the trace interval is several orders of magnitude greater than in trace eyeblink conditioning. Inactivation of $\mathrm{mPFC}$ during trace fear conditioning impaired freezing fear responses $24 \mathrm{~h}$ later. Disruption of prefrontal activity was temporary, as these rats were able to learn trace fear conditioning when retrained several days later. The importance of intact prefrontal function during trace, but not delay conditioning, suggests that prefrontal activity may be necessary for the association of the CS and UCS when they are separated in time. 


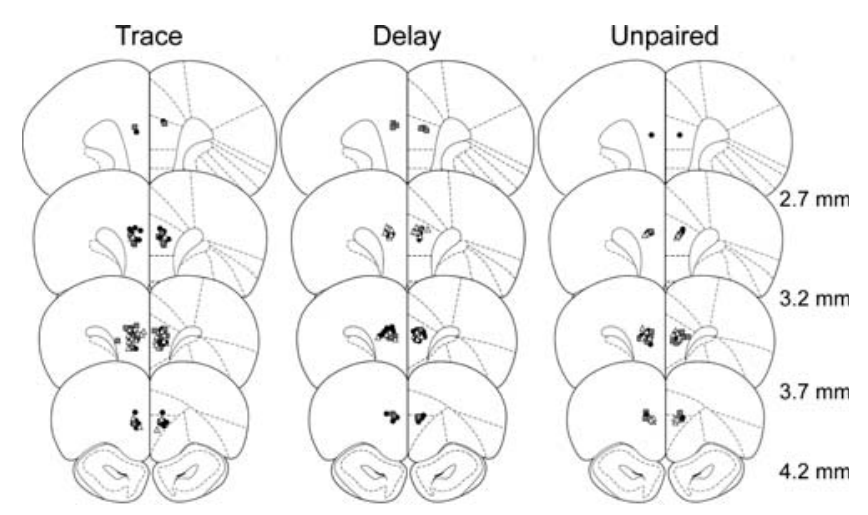

Figure 5. Placement of bilateral injection cannulae in prelimbic mPFC. Symbols on each coronal diagram show the placement of injector tips in the prelimbic for rats that received infusions of saline (black circles), muscimol (gray squares), or APV (open triangles) in the trace, delay, and unpaired conditioning groups. Coronal diagrams are shown for four levels anterior to bregma. (Diagrams are adapted from Paxinos and Watson 1998, and reprinted with permission of Elsevier (C) 1998.)

Activity in the prefrontal cortex may be necessary to bridge the temporal gap between CS and UCS in trace fear conditioning. In order for the CS to be associated with the UCS during temporal learning such as trace conditioning, a representation of the CS may need to be present when the UCS is delivered (Wallenstein et al. 1998; Rodriguez and Levy 2001; Mongillo et al. 2003; Reutimann et al. 2004). One function of the mPFC is to maintain cue-related information in working memory for seconds or minutes in order to perform task-relevant responses (Fuster 1989). Cue-induced sustained increases in prefrontal single-neuron firing have been observed during delay periods in a number of working memory tasks in rats and primates (Fuster 1973; Funahashi et al. 1989; Sawaguchi and Yamane 1999; Chang et al. 2002). Patterns of tonic firing have also been observed during trace fear conditioning. Individual neurons recorded from prelimbic mPFC during trace fear conditioning show sustained increases during a short, 3-sec trace interval (Baeg et al. 2001) or during a long, 20-sec trace interval (Gilmartin and McEchron 2005). In contrast, prelimbic units recorded from unpaired control animals did not exhibit the same degree of post-CS sustaining activity, suggesting that trace interval firing was specific to the CS-trace-UCS association (Gilmartin and McEchron 2005). Prefrontal activity during the trace interval has also been demonstrated in human subjects. Blood-oxygen level-dependent (BOLD) activity measured by functional magnetic resonance imaging (fMRI) in prefrontal regions in humans is observed during the trace interval period in trace but not during a comparable period in delay fear conditioning (Knight et al. 2004). These findings in rats and humans suggest that the prefrontal cortex may provide a bridging role during trace conditioning, but they do not demonstrate a necessity for this activity. The present findings demonstrate that cellular activity in mPFC is critical to the acquisition of trace fear conditioning. Rats with temporarily inhibited excitatory activity in the mPFC during trace fear conditioning showed little to no fear responses to the CS $24 \mathrm{~h}$ later, similar to control rats that received unpaired nonassociative presentations of the CS and US. Although this study did not directly test a requirement for CS-related sustaining activity during associative learning, a recent study by Mauk and colleagues (Kalmbach et al. 2009) provides evidence that cortical sustained activity is essential for trace conditioning. Using stimulus parameters of trace eyeblink conditioning, they showed that sustained activity in cortical inputs to the cerebellum is necessary for the CS-UCS association
(Kalmbach et al. 2009). Prelimbic mPFC may be the cortical structure providing these sustained CS-related responses during the trace interval in trace conditioning tasks.

The requirement of prefrontal neuronal activity for trace fear conditioning is consistent with the well-established role for this structure in working memory. However, recent work has demonstrated a role for prefrontal plasticity in long-term memory (i.e., $24 \mathrm{~h}$ or longer) (e.g., Jung et al. 2008) and there is some evidence that mPFC may contribute to the long-term stability of trace fear memories. Pre- or post-training disruption of MAPK intracellular signaling or post-training inhibition of protein synthesis in the mPFC impairs the formation of trace fear memory (Runyan et al. 2004; Blum et al. 2006b). The mechanisms during training that may contribute to long-term changes in prefrontal plasticity are poorly understood. This study tested whether NMDAR activation during the acquisition period is important for the formation of long-term trace fear memory. NMDAR-dependent mechanisms have been shown to be critical for several forms of LTP in vitro, a cellular model of long-term memory (Bliss and Collingridge 1993). NMDAR neurotransmission is also critical for memory formation in vivo for a number of learning paradigms, including in the amygdala for delay fear conditioning and in the hippocampus for trace fear conditioning (Kim et al. 1992; Maren et al. 1996; Huerta et al. 2000; Wanisch et al. 2005). Our results show that NMDAR neurotransmission is also necessary in the mPFC for trace fear conditioning. This finding corroborates similar results from trace eyeblink conditioning: Daily blockade of prefrontal NMDARs during trace eyeblink conditioning impaired the acquisition of eyeblink conditional responses (Takehara-Nishiuchi et al. 2005). Given that induction of LTP at hippocampal-prefrontal synapses requires NMDARs (Jay et al. 1995), it is possible that hippocampally driven activation of these receptors in prelimbic mPFC triggers intracellular signaling and the subsequent synthesis of new protein important for cellular plasticity. It is important to note that a requirement for NMDAR neurotransmission does not confirm prefrontal plasticity in the formation of trace fear conditioning. $\mathrm{Ca}^{2+}$ signaling via activation of NMDARs and L-type calcium channels has been proposed to provide a mechanism supporting persistent neuronal activity similar to that observed in the mPFC during working memory tasks (Lisman et al. 1998; Egorov et al. 2002; Fransen et al. 2006). Because NMDAR activation is sensitive to postsynaptic voltage changes, NMDAR activation may serve to select a specific subset of neurons to maintain persistent activity in working memory (Lisman et al 1998).

Disruption of cellular activity in prelimbic mPFC in this study impaired contextual fear conditioning in the trace and delay groups. Contextual fear responses are acquired simultaneously with auditory CS fear responses during trace or delay fear conditioning, and retention of contextual fear is tested in the original training environment in the absence of auditory cues. Acquiring fear to the configural properties of the training environment is dependent on the hippocampus, and it is unclear what role the mPFC plays in the acquisition and consolidation of these fear responses. Electrolytic lesions of the ventral mPFC (including ventral prelimbic, infralimbic, and caudal medial orbital cortex) administered prior to delay fear conditioning failed to impair context fear acquisition (Morgan et al. 1993), and similar lesions to the dorsal mPFC (including prelimbic and anterior cingulate $[\mathrm{ACg}]$ ) augmented fear responses to the context (Morgan and LeDoux 1995). We found that disruption of prelimbic mPFC activity during training impairs context fear conditioning in trace- and delay-conditioned rats, consistent with more recent work on this issue. Similar to our findings, Quinn and colleagues (2008) observed contextual impairments after permanent post-training lesions of mPFC following trace and delay conditioning. We also 
show that blockade of NMDAR-mediated signaling in the prelimbic mPFC impairs contextual fear responses acquired during paired conditioning. This result is consistent with a recent study in which selective blockade of the NR2B subunit of the NMDA receptor in the ACg mPFC impaired both cingulate LTP and the formation of contextual fear memory during a one-trial auditory fear conditioning session (Zhao et al. 2005). Together, these findings suggest that dorsal areas of $\mathrm{mPFC}$ contribute to the formation of contextual fear memory.

These findings demonstrate a role for mPFC in the formation of contextual fear memory when it is acquired simultaneously with fear to a discrete auditory cue during paired auditory fear conditioning. We did not find impairments in contextual fear retention in the unpaired group, where the context, but not the auditory CS, predicts the UCS. This suggests that the MPFC may be necessary to associate the training context with the UCS when the context has a weaker predictive relationship with the UCS relative to the foreground auditory CS-UCS association. When the context-UCS association is in the foreground, as in unpaired training, prefrontal activity may not be required. However, other regions of the MPFC may be important for foreground contextual fear learning. Blockade of NMDA receptors in the ventral $\mathrm{mPFC}$ (predominantly infralimbic) immediately before a context retention test impaired the expression of a foreground contextual memory (Resstel et al. 2008). Clearly, more work is needed to determine the circumstances underlying a prefrontal role in the acquisition and expression of contextual fear conditioning.

Our findings lend further support for a prefrontal role in the initial formation of memory, expanding upon known roles for this structure in working memory, extinction, fear expression, and systems consolidation (Takehara et al. 2003; Frankland et al. 2004; Santini et al. 2004; Corcoran and Quirk 2007; Jung et al. 2008). Much of the previous work on the contribution of frontal regions to long-term memory demonstrated that anterior cingulate and prelimbic mPFC participates in the systems consolidation of some memories-the gradual transition of memory from dependence on subcortical structures to cortical sites (Frankland and Bontempi 2005). Permanent lesions or temporary inactivation of mPFC, including ACg or PL, impair contextual fear and spatial memories when administered weeks after training, but not when administered 1 or 2 days after training (Frankland et al. 2004; Teixeira et al. 2006; Ding et al. 2008). Similar results have been observed for trace eyeblink and trace fear paradigms (Takehara et al. 2003; Quinn et al. 2008; but see Blum et al. 2006a). These manipulations occur post-training, and the mPFC is intact during the initial acquisition. Our findings demonstrate a need for an intact prelimbic mPFC for the initial formation of memory. Taken together, these two lines of evidence suggest that the PL mPFC may participate in multiple phases of memory formation. The mPFC is necessary for the encoding of trace fear conditioning and may store information about the CS-UCS association; however, the information initially stored in this structure may not be critical to the expression of the memory when the memory is still recent. As the memory ages, a separate mechanism may engage to consolidate the memory in cortical circuits. Trace fear conditioning may provide a useful means to examine multiple phases of prefrontal mechanisms of memory.

In conclusion, acquisition of hippocampus-dependent trace fear conditioning requires cellular activity in mPFC. Prefrontal NMDAR-mediated signaling is necessary for the association of the CS and UCS when they are separated by a 20 -sec trace interval. Given that LTP at hippocampus-prelimbic synapses requires NMDAR activation (Jay et al. 1995), it is likely that the hippocampus and mPFC interact to encode the CS-trace-UCS association. Future investigations of the cooperative nature of hippocampal and prefrontal mechanisms of acquisition and consolidation in trace fear conditioning will provide new insights into the systems-level control of memory formation.

\section{Materials and Methods}

\section{Subjects and surgery}

Surgery was performed on a total of 120 adult male Long-Evans rats in this study (325-400 g; Harlan, IN). All rats were housed individually and received food and water ad libitum. All surgical procedures were in accordance with the National Institutes of Health and the University of Wisconsin-Milwaukee Institutional Animal Care and Use Committee. Rats were anesthetized with either $2.5 \mathrm{mg} / \mathrm{kg}$ sodium pentobarbital $(n=44)$ or $1 \%-$ $2 \%$ isoflurane in $100 \% \mathrm{O}_{2}(n=76)$ during surgery. Each rat was positioned in a stereotaxic frame and the skull was exposed. Two skull screws (\#0-80-02-M, Small Parts, Inc.) were inserted $\sim 1 \mathrm{~mm}$ into the skull to anchor the final acrylic cement head assembly. Holes of 4-mm diameter were drilled in the skull above the left and right medial prefrontal cortex. Stainless steel guide cannulae (26 ga; Plastics One, Inc.) were stereotaxically lowered to the dorsal border of the prelimbic area of the $\mathrm{mPFC}$, bilaterally at a $15^{\circ}$ angle to vertical $(\mathrm{AP}+3.2 \mathrm{~mm} ; \mathrm{ML} \pm 1.6 \mathrm{~mm}$; $\mathrm{DV}$ $-3.2 \mathrm{~mm}$ from the skull). Acrylic cement was used to secure the cannulae to the skull and 33-ga dummy cannulae were screwed into the guide cannulae to prevent clogging.

\section{Infusion procedure}

Following recovery from surgery, rats received $3 \mathrm{~d}$ of acclimation to transport from their home cages to the procedure rooms. During this time, the rats were also acclimated to gentle restraint in a towel and to the sound of the infusion pump that would be used for intracranial injections. No infusions were delivered during these acclimation sessions. On the day of conditioning, rats received bilateral infusions $(0.3 \mu \mathrm{L} /$ side $)$ of the $\mathrm{GABA}_{\mathrm{A}}$ agonist muscimol $(1 \mu \mathrm{g} / \mu \mathrm{L} ;$ 5-aminomethyl-3-hydroxyisoxazole, MP Biomedicals), the NMDA receptor antagonist DL-APV $(10 \mu \mathrm{g} / \mu \mathrm{L}$; Tocris), or sterile saline vehicle $15 \mathrm{~min}$ prior to training. Drug or vehicle was infused at a rate of $0.5 \mu \mathrm{L} / \mathrm{min}$ through 33-ga injection cannulae, which extended $0.5 \mathrm{~mm}$ below the end of the guide cannulae. Injectors were left in place for $90 \mathrm{sec}$ following the completion of the infusion to allow for diffusion of the drug or vehicle away from the cannulae. Rats were returned to their home cages immediately after the infusion procedure. The time point for infusion was based on reports that muscimol and APV take effect within minutes of infusion and may be cleared from the system within 2-3 h post-infusion (Martin 1991; Steele and Morris 1999). Training is complete within $1 \mathrm{~h}$ of drug infusion.

\section{Conditioning}

Fifteen minutes after the end of the infusion, rats were placed in the conditioning chambers and each received six trials of paired trace $(n=48)$ or delay $(n=40)$ fear conditioning, or 12 trials of unpaired control training $(n=32)$ following a 6-min baseline period. Each trace fear conditioning trial consisted of a 10 -sec white noise conditional stimulus (CS; $72 \mathrm{~dB}$ ) and a 1-s footshock unconditional stimulus (UCS; $1 \mathrm{~mA}$ ) separated by an empty $20-\mathrm{s}$ trace interval. The intertrial interval (ITI) for this session was $240 \pm 20 \mathrm{sec}$. Delay fear conditioning (ITI $=260 \pm 20 \mathrm{sec}$ ) was the same as trace fear conditioning, except that the UCS was delivered at CS offset. Unpaired control training $(120 \pm 20 \mathrm{sec})$ consisted of the same number of CSs and UCSs as the paired training, but the UCS and CS were presented explicitly unpaired. This training served as a control for nonassociative responding to the white noise CS. All training sessions lasted $33 \mathrm{~min}$. Conditioning occurred in a set of four Plexiglas and stainless steel conditioning chambers (internal dimensions: $21 \times 28 \times 21 \mathrm{~cm}$ ), each housed in a sound attenuating outer chamber and illuminated with a white incandescent house lamp. Ventilation fans 
in each outer chamber provided 63-65-dB background noise and the white noise CS was delivered through a speaker centered in the end of each conditioning chamber. Stainless steel bars (4-mm diameter, spaced $12 \mathrm{~mm}$ apart) on the floor of each chamber served to deliver the footshock UCS. The chambers were cleaned and wiped down with 5\% ammonium hydroxide solution between each set of rats.

Twenty-four hours after training, rats received a single CS retention test in a novel chamber and a single Context retention test in the original training chamber. The CS retention test consisted of a 6-min baseline period, followed by a 5-min continuous white-noise CS presentation and a 4-min post-CS period. The Context retention test consisted of 15 -min reexposure to the training chamber. CS and Context tests were separated by $4 \mathrm{~h}$ and counterbalanced within each group. Novel testing chambers used for the CS test (internal dimensions: $20.5 \times 26.5 \times 21 \mathrm{~cm}$ ) were each housed in a sound-attenuating outer chamber with $58-60-\mathrm{dB}$ background noise. These chambers were in a separate room and differed from the training chambers in illumination (infrared house lamp), texture (solid floor), and odor (5\% acetic acid solution).

Twenty-four hours after testing, trace and delay rats were retrained in the absence of drug. Retraining and testing procedures were identical to the original training and testing procedures for each rat. Whenever possible, all rats were retrained; however, because of large group sizes, a random selection of rats was not retrained.

\section{Analyses}

Freezing, the cessation of all movement except that needed for respiration, was used as the measure of conditional fear during all training and testing sessions (Fanselow and Bolles 1979). Freezing was scored automatically in real-time using the FreezeScan 1.0 detection software (Clever Sys, Inc.). All statistical analyses were performed with Statistica version 9 (Statsoft, Inc.). Each training group was analyzed separately and drug differences in freezing were analyzed using one-way ANOVAs (immediate post-acquisition; context retention) or mixed-model ANOVAs with repeated measures (CS retention), which included the following factors: a repeated measure of Period (Baseline, CS, and Post-CS periods) and a between factor of Drug (saline, muscimol, and APV). Fisher LSD post-hoc tests were used to test the significance of mean differences. An $\alpha$ level of 0.05 was required for significance in all analyses. Three rats in the trace muscimol group were flagged as statistical outliers using a standard outlier test in Statistica based on percentiles and were excluded from analyses. These rats showed near ceiling levels of freezing during the CS retention test, levels that were greater than those of most control rats. The remaining muscimol-infused rats all showed consistently low CS freezing levels below 35\%. The median freezing without these three outliers is $16.52 \%$. While we cannot rule out the possibility that these three rats received a complete infusion of muscimol and were able to learn the CS-UCS association, these rats are clearly different from the majority of the muscimol-infused trace group.

\section{Histology}

At the end of the experiment, rats were deeply anesthetized with $5 \%$ isoflurane in $100 \% \mathrm{O}_{2}$, transcardially perfused with $0.9 \%$ saline followed by $10 \%$ buffered formalin, and the brains were placed in a $10 \%$ formalin solution (in $0.9 \%$ saline). Brains were transferred to a $20 \%$ sucrose/formalin solution prior to processing for histology. Brains were then frozen, sectioned coronally, mounted on glass slides, and stained with cresyl violet.

\section{Acknowledgments}

This research was supported by the National Institute of Mental Health (NIMH) grant R01MH069558 to F.J.H. and NIMH grant F32MH083422 to M.R.G. We thank Janine Kwapis and Mary Lonergan for assistance with the drug microinfusions.

\section{References}

Allen TA, Narayanan NS, Kholodar-Smith DB, Zhao Y, Laubach M, Brown TH. 2008. Imaging the spread of reversible brain inactivations using fluorescent muscimol. I Neurosci Methods 171: 30-38.

Baeg EH, Kim YB, Jang J, Kim HT, Mook-Jung I, Jung MW. 2001. Fast spiking and regular spiking neural correlates of fear conditioning in the medial prefrontal cortex of the rat. Cereb Cortex 11: 441-451.

Bliss TV, Collingridge GL. 1993. A synaptic model of memory: Long-term potentiation in the hippocampus. Nature 361: $31-39$

Blum S, Hebert AE, Dash PK. 2006a. A role for the prefrontal cortex in recall of recent and remote memories. Neuroreport 17: 341-344.

Blum S, Runyan JD, Dash PK. 2006b. Inhibition of prefrontal protein synthesis following recall does not disrupt memory for trace fear conditioning. BMC Neurosci 7: 67. doi: 10.1186/1471-2202-7-67.

Blumenfeld RS, Ranganath C. 2006. Dorsolateral prefrontal cortex promotes long-term memory formation through its role in working memory organization. J Neurosci 26: 916-925.

Chang JY, Chen L, Luo F, Shi LH, Woodward DJ. 2002. Neuronal responses in the frontal cortico-basal ganglia system during delayed matching-to-sample task: Ensemble recording in freely moving rats. Exp Brain Res 142: 67-80.

Corcoran KA, Quirk GJ. 2007. Activity in prelimbic cortex is necessary for the expression of learned, but not innate, fears. J Neurosci 27: 840-844.

Ding HK, Teixeira CM, Frankland PW. 2008. Inactivation of the anterior cingulate cortex blocks expression of remote, but not recent, conditioned taste aversion memory. Learn Mem 15: 290-293.

Egorov AV, Hamam BN, Fransen E, Hasselmo ME, Alonso AA. 2002. Graded persistent activity in entorhinal cortex neurons. Nature 420: $173-178$.

Fanselow MS, Bolles RC. 1979. Naloxone and shock-elicited freezing in the rat. J Comp Physiol Psychol 93: 736-744.

Frankland PW, Bontempi B. 2005. The organization of recent and remote memories. Nat Rev Neurosci 6: 119-130.

Frankland PW, Bontempi B, Talton LE, Kaczmarek L, Silva AJ. 2004. The involvement of the anterior cingulate cortex in remote contextual fear memory. Science 304: 881-883.

Fransen E, Tahvildari B, Egorov AV, Hasselmo ME, Alonso AA. 2006. Mechanism of graded persistent cellular activity of entorhinal cortex layer v neurons. Neuron 49: 735-746.

Funahashi S, Bruce CJ, Goldman-Rakic PS. 1989. Mnemonic coding of visual space in the monkey's dorsolateral prefrontal cortex. $J$ Neurophysiol 61: 331-349.

Fuster JM. 1973. Unit activity in prefrontal cortex during delayed-response performance: Neuronal correlates of transient memory. J Neurophysiol 36: $61-78$.

Fuster JM. 1989. The prefrontal cortex, 2nd ed. Raven Press, New York.

Fuster JM. 2001. The prefrontal cortex-an update: Time is of the essence. Neuron 30: 319-333.

Gilmartin MR, McEchron MD. 2005. Single neurons in the medial prefrontal cortex of the rat exhibit tonic and phasic coding during trace fear conditioning. Behav Neurosci 119: 1496-1510.

Huerta PT, Sun LD, Wilson MA, Tonegawa S. 2000. Formation of temporal memory requires NMDA receptors within CA1 pyramidal neurons. Neuron 25: 473-480.

Jay TM, Burette F, Laroche S. 1995. NMDA receptor-dependent long-term potentiation in the hippocampal afferent fibre system to the prefrontal cortex in the rat. Eur J Neurosci 7: 247-250.

Jung MW, Baeg EH, Kim MJ, Kim YB, Kim JJ. 2008. Plasticity and memory in the prefrontal cortex. Rev Neurosci 19: 29-46.

Kalmbach BE, Ohyama T, Kreider JC, Riusech F, Mauk MD. 2009. Interactions between prefrontal cortex and cerebellum revealed by trace eyelid conditioning. Learn Mem 16: 86-95.

Kim JJ, Fanselow MS, DeCola JP, Landeira-Fernandez J. 1992. Selective impairment of long-term but not short-term conditional fear by the N-methyl-D-aspartate antagonist APV. Behav Neurosci 106: 591-596.

Knight DC, Cheng DT, Smith CN, Stein EA, Helmstetter FJ. 2004. Neural substrates mediating human delay and trace fear conditioning. $J$ Neurosci 24: 218-228.

Kronforst-Collins MA, Disterhoft JF. 1998. Lesions of the caudal area of rabbit medial prefrontal cortex impair trace eyeblink conditioning. Neurobiol Learn Mem 69: 147-162.

LaLumiere RT, Niehoff KE, Kalivas PW. 2010. The infralimbic cortex regulates the consolidation of extinction after cocaine self-administration. Learn Mem 17: 168-175.

Laroche S, Jay TM, Thierry AM. 1990. Long-term potentiation in the prefrontal cortex following stimulation of the hippocampal CA1/ subicular region. Neurosci Lett 114: 184-190.

Lee I, Kesner RP. 2002. Differential contribution of NMDA receptors in hippocampal subregions to spatial working memory. Nat Neurosci 5: $162-168$

Lisman JE, Fellous JM, Wang XJ. 1998. A role for NMDA-receptor channels in working memory. Nat Neurosci 1: 273-275. 
Maren S, Aharonov G, Stote DL, Fanselow MS. 1996. N-methyl-D-aspartate receptors in the basolateral amygdala are required for both acquisition and expression of conditional fear in rats. Behav Neurosci 110: 1365-1374.

Martin JH. 1991. Autoradiographic estimation of the extent of reversible inactivation produced by microinjection of lidocaine and muscimol in the rat. Neurosci Lett 127: 160-164.

McEchron MD, Bouwmeester H, Tseng W, Weiss C, Disterhoft JF. 1998. Hippocampectomy disrupts auditory trace fear conditioning and contextual fear conditioning in the rat. Hippocampus 8: 638-646.

McLaughlin J, Skaggs H, Churchwell J, Powell DA. 2002. Medial prefrontal cortex and Pavlovian conditioning: Trace versus delay conditioning. Behav Neurosci 116: 37-47.

Mongillo G, Amit DJ, Brunel N. 2003. Retrospective and prospective persistent activity induced by Hebbian learning in a recurrent cortical network. Eur J Neurosci 18: 2011-2024.

Morgan MA, LeDoux JE. 1995. Differential contribution of dorsal and ventral medial prefrontal cortex to the acquisition and extinction of conditioned fear in rats. Behav Neurosci 109: 681-688.

Morgan MA, Romanski LM, LeDoux JE. 1993. Extinction of emotional learning: Contribution of medial prefrontal cortex. Neurosci Lett 163: $109-113$.

Morgan MA, Schulkin J, LeDoux JE. 2003. Ventral medial prefrontal cortex and emotional perseveration: The memory for prior extinction training. Behav Brain Res 146: 121-130.

Quinn JJ, Loya F, Ma QD, Fanselow MS. 2005. Dorsal hippocampus NMDA receptors differentially mediate trace and contextual fear conditioning. Hippocampus 15: 665-674.

Quinn JJ, Ma QD, Tinsley MR, Koch C, Fanselow MS. 2008. Inverse temporal contributions of the dorsal hippocampus and medial prefrontal cortex to the expression of long-term fear memories. Learn Mem 15: 368-372.

Resstel LB, Correa FM, Guimaraes FS. 2008. The expression of contextual fear conditioning involves activation of an NMDA receptor-nitric oxide pathway in the medial prefrontal cortex. Cereb Cortex 18: 2027-2035.

Reutimann J, Yakovlev V, Fusi S, Senn W. 2004. Climbing neuronal activity as an event-based cortical representation of time. J Neurosci 24: $3295-3303$.

Reymann KG, Matthies HK, Schulzeck K, Matthies H. 1989. $\mathrm{N}$-methyl-D-aspartate receptor activation is required for the induction of both early and late phases of long-term potentiation in rat hippocampal slices. Neurosci Lett 96: 96-101.

Rodriguez P, Levy WB. 2001. A model of hippocampal activity in trace conditioning: Where's the trace? Behav Neurosci 115: 1224-1238.

Runyan JD, Moore AN, Dash PK. 2004. A role for prefrontal cortex in memory storage for trace fear conditioning. J Neurosci 24: $1288-1295$.
Sacchetti B, Baldi E, Lorenzini CA, Bucherelli C. 2002. Differential contribution of some cortical sites to the formation of memory traces supporting fear conditioning. Exp Brain Res 146: 223-232.

Santini E, Ge H, Ren K, Pena de Ortiz S, Quirk GJ. 2004. Consolidation of fear extinction requires protein synthesis in the medial prefrontal cortex. J Neurosci 24: 5704-5710.

Sawaguchi T, Yamane I. 1999. Properties of delay-period neuronal activity in the monkey dorsolateral prefrontal cortex during a spatial delayed matching-to-sample task. J Neurophysiol 82: 2070-2080.

Solomon PR, Vander Schaaf ER, Thompson RF, Weisz DJ. 1986. Hippocampus and trace conditioning of the rabbit's classically conditioned nictitating membrane response. Behav Neurosci 100: $729-744$.

Steele RJ, Morris RG. 1999. Delay-dependent impairment of a matching-to-place task with chronic and intrahippocampal infusion of the NMDA-antagonist D-AP5. Hippocampus 9: 118-136.

Takehara-Nishiuchi K, Kawahara S, Kirino Y. 2005. NMDA receptor-dependent processes in the medial prefrontal cortex are important for acquisition and the early stage of consolidation during trace, but not delay eyeblink conditioning. Learn Mem 12: $606-614$.

Takehara K, Kawahara S, Kirino Y. 2003. Time-dependent reorganization of the brain components underlying memory retention in trace eyeblink conditioning. J Neurosci 23: 9897-9905.

Teixeira CM, Pomedli SR, Maei HR, Kee N, Frankland PW. 2006. Involvement of the anterior cingulate cortex in the expression of remote spatial memory. J Neurosci 26: 7555-7564.

Tonegawa S, Tsien JZ, McHugh TJ, Huerta P, Blum KI, Wilson MA. 1996. Hippocampal CA1-region-restricted knockout of NMDAR1 gene disrupts synaptic plasticity, place fields, and spatial learning. Cold Spring Harb Symp Quant Biol 61: 225-238.

Wallenstein GV, Eichenbaum H, Hasselmo ME. 1998. The hippocampus as an associator of discontiguous events. Trends Neurosci 21: $317-323$.

Wanisch K, Tang J, Mederer A, Wotjak CT. 2005. Trace fear conditioning depends on NMDA receptor activation and protein synthesis within the dorsal hippocampus of mice. Behav Brain Res 157: $63-69$.

Weible AP, McEchron MD, Disterhoft JF. 2000. Cortical involvement in acquisition and extinction of trace eyeblink conditioning. Behav Neurosci 114: 1058-1067.

Zhao MG, Toyoda H, Lee YS, Wu LJ, Ko SW, Zhang XH, Jia Y, Shum F, Xu H, Li BM, et al. 2005. Roles of NMDA NR2B subtype receptor in prefrontal long-term potentiation and contextual fear memory. Neuron 47: $859-872$.

Received August 17, 2009; accepted in revised form April 22, 2010. 


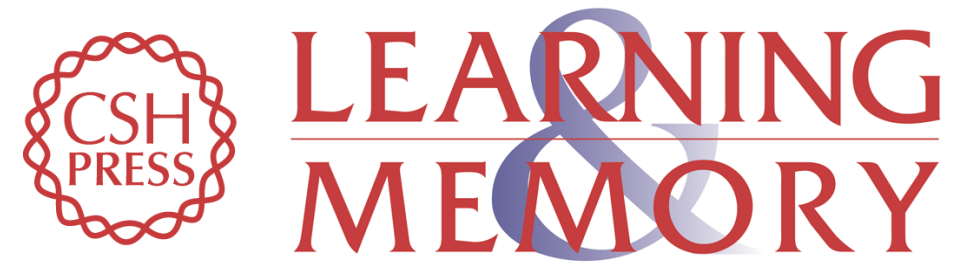

\section{Trace and contextual fear conditioning require neural activity and NMDA receptor-dependent transmission in the medial prefrontal cortex}

Marieke R. Gilmartin and Fred J. Helmstetter

Learn. Mem. 2010, 17:

Access the most recent version at doi: $10.1101 / \mathrm{lm} .1597410$

References This article cites 57 articles, 15 of which can be accessed free at: http://learnmem.cshlp.org/content/17/6/289.full.html\#ref-list-1

License

Email Alerting

Receive free email alerts when new articles cite this article - sign up in the box at the Service top right corner of the article or click here. 